\title{
Haag's theorem, apparent inconsistency, and the empirical adequacy of quantum field theory
}

\author{
Michael E. Miller ${ }^{\dagger}$
}

\begin{abstract}
Haag's theorem has been interpreted as establishing that quantum field theory cannot consistently represent interacting fields. Earman and Fraser have clarified how it is possible to give mathematically consistent calculations in scattering theory despite the theorem. However, their analysis does not fully address the worry raised by the result. In particular, I argue that their approach fails to be a complete explanation of why Haag's theorem does not undermine claims about the empirical adequacy of particular quantum field theories. I then show that such empirical adequacy claims are protected from Haag's result by the techniques that are required to obtain theoretical predictions for realistic experimental observables. I conclude by showing how Haag's theorem is illustrative of a general tension between the foundational significance of results that can be obtained in perturbation theory and non-perturbative characterizations of the content of quantum field theory.
\end{abstract}

1. Introduction. Despite the often noted empirical successes of the Standard Model of particle physics, the quantum field theories on which it is based have been shown to be mathematically questionable in a number of respects. One such mathematical problem is captured by a result originally proved by Haag, and subsequently generalized by Hall and Wightman. ${ }^{1}$ Haag's theorem has received significant attention because it raises the specter of inconsistency in the context of interacting quantum field theories. For example, Teller claims that because of the theorem "... there appears to be no known consistent formalism within which interacting quantum field theory can be expressed" (Teller 1995, p. 115). ${ }^{2}$ If this claim was correct, then it would be difficult to understand how so much empirical evidence for the interacting quantum field theories that make up the Standard Model has been accumulated. Roughly, the theorem shows that the assumptions required to form the interaction picture in which scattering theory calculations are carried out are consistent only in the case of non-interacting theories. In this sense, the theorem does establish the inconsistency of a set of assumptions that are sometimes simultaneously assumed to hold in interacting quantum field theories. In this paper I show why this does not undermine the empirical adequacy claims that are taken to support the quantum field theories that make up the Standard Model.

\footnotetext{
* Department of History and Philosophy of Science, University of Pittsburgh

Email: mem208@pitt.edu

${ }^{1}$ Haag's original result was proved in (Haag 1955) and the Hall and Wightman generalization can be found in (Hall and Wightman 1957). The complex historical development of the theorem has been recounted by Lupher (Lupher 2005).

${ }^{2}$ They also note that similar claims can be found in (Barton 1963, p. 157), (Huggett and Weingard 1994, p. 376), and (Sklar 2000, p. 28).
} 
Earman and Fraser have made progress in this direction by arguing that previous attempts to articulate the foundational significance of the theorem tend toward "overstatement" and even "hyperventilation" (Earman and Fraser 2006, p. 305, p. 323). Their analysis leads them to three central conclusions. First, it emphasizes the importance of unitarily inequivalent representations of the canonical commutation relations, whose existence in quantum field theory distinguish it from non-relativistic quantum mechanics. Second, it makes it clear that non-Fock representations have an important role to play in quantum field theory. Finally they claim that the theorem undermines the standard interaction picture formalism and the approaches to scattering theory that depend on it. In particular they claim that "... while Haag's theorem does not show that no quantum field theory exists which differs from a free field theory, it does pose problems for some of the techniques used in textbook physics for extracting physical predictions from the theory" (Earman and Fraser 2006, p. 306). They diagnose the strong reaction to the theorem in the literature as referring to this fact (Earman and Fraser 2006, pp. 306-307). While I agree with the first two conclusions that they draw concerning the importance of the theorem, this paper provides further analysis of the third. This further analysis is necessary in order to properly understand how Haag's theorem bears on the issues of consistency and empirical adequacy for quantum field theory. ${ }^{3}$ The textbook calculations they refer to have played an important role in establishing the empirical adequacy of particular models of the theory. If Haag's theorem shows such calculations to be predicated on an inconsistent set of assumptions, then those empirical adequacy claims are unreliable.

Scattering theory calculations are the basis for comparison between quantum field theories and experiments, and thus some explanation for why field theoretic scattering theory matches empirical data, despite Haag's result, is required. In order to explain this success Earman and Fraser appeal to a mathematically rigorous formalism for scattering theory due to Haag and Ruelle which circumvents Haag's theorem. While this formalism does demonstrate that scattering theory can be formalized in a mathematically consistent manner, the existence of such a formalism does not fully resolve the worry raised by Haag's theorem because it does not explain why theoretical predictions for realistic experimental observables give empirically adequate results. There is, however, a clear reason why such theoretical calculations are not undermined by Haag's theorem; namely, in those cases where the interaction picture is employed the calculational techniques that are required to extract predictions from empirically adequate field theories violate some of

\footnotetext{
${ }^{3}$ Earman and Fraser agree as they note that their analysis leaves "... unfinished business in explaining why perturbation theory works as well as it does" (Earman and Fraser 2006, pp. 306-307).
} 
the assumptions required to prove the theorem. In other cases, the theoretical calculations that are used to compare to experiments simply do not use the interaction picture in any way. It is these facts that explain why Haag's theorem does not directly undermine claims about the empirical adequacy of quantum field theories.

This situation shows that Haag's theorem is illustrative of a general tension which exists in much of the literature that is engaged with the philosophical appraisal of the foundations of quantum field theory. It is often unclear how fully mathematically rigorous models inform claims about the actual world because they are defined in reduced spacetime dimension or do not represent realistic interactions. Conversely, it is often not obvious whether or not one should assign interpretive significance to the changes to the mathematical formalism that are required to render calculations of physical observables well-defined. A complete understanding of the significance of Haag's theorem requires analysis of how it bears on both of these problems. I argue that Haag's theorem should be understood as a constraint on the nature of the relation between results obtained in perturbation theory and exact non-perturbative characterizations of quantum field theories, in the sense that it rules out one particular method for forming the infrared limit of a fully regularized theory.

My argument proceeds as follows. The second section briefly introduces the interaction picture formalism for scattering theory and explains how Haag's theorem shows that it is predicated on an inconsistent set of assumptions. In the third section I consider Earman and Fraser's explanation of the success of scattering theory and show that it does not resolve the worry that empirical adequacy claims are undermined by the result. The fourth section shows how the calculational techniques required to obtain empirical predictions avoid Haag's theorem by considering examples of calculations in quantum electrodynamics and quantum chromodynamics. In the concluding section I address how Haag's theorem bears on the relation between perturbative calculations and non-perturbative structure.

2. Haag's theorem and the interaction picture. Haag's theorem undermines the interaction picture and the standard approach to scattering theory. It does so by showing that the assumptions required to formulate the interaction picture are inconsistent with the presence of a non-trivial interaction in the theory. Thus, when the interaction picture is used for calculations in theories like quantum electrodynamics which contain interactions, the calculations possess an apparent mathematical inconsistency. Furthermore, there is good reason to worry that this renders empirical adequacy claims for particular field theories unreliable. Scattering theory provides the critical connection between a quantum field theory and experimental observables such as cross-sections. Empirical adequacy claims for quantum field 
theories are based on the agreement between cross-sections calculated with scattering theory and cross-sections observed in experiments at particle accelerators. When the quantum field theories of the Standard Model are used in such calculations they yield results that closely match the observed values for the quantities. Much of the direct evidence for the empirical adequacy of the Standard Model is derived, either directly or indirectly, from comparisons of this sort. In some cases, these theoretical calculations use the interaction picture formalism which is undermined by Haag's theorem. In this way, the theorem seems to show that the formalism that has produced what can be counted among the most precisely confirmed predictions of any physical theory is mathematically inconsistent.

The interaction picture is an intermediate between the Schrödinger picture, in which states evolve in time under the full Hamiltonian and operators are stationary, and the Heisenberg picture, in which states are stationary and operators evolve under the full Hamiltonian. ${ }^{4}$ States and operators in the interaction picture are given the subscript, $I$. The time evolution of operators in the Heisenberg picture is determined by the Heisenberg equation of motion: $\partial O_{H}(t) / \partial t=-i\left[O_{H}(t), H\right]$. Operators in the Schrödinger picture are related to the Heisenberg picture by the transformation, $O_{S}=e^{-i H t} O_{H}(t) e^{i H t}$, and the states are related by, $\psi_{S}(t)=e^{-i H t} \psi_{H}$. These transformations leave the matrix elements of corresponding operators invariant,

$$
\begin{aligned}
{ }_{H}\left\langle\psi\left|O_{H}(t)\right| \phi\right\rangle_{H} & ={ }_{H}\left\langle\psi\left|e^{i H t} e^{-i H t} O_{H}(t) e^{i H t} e^{-i H t}\right| \psi\right\rangle_{H} \\
& ={ }_{S}\left\langle\psi(t)\left|O_{S}\right| \phi(t)\right\rangle_{S},
\end{aligned}
$$

and in this sense they are empirically equivalent. The interaction picture is formed by writing the full Hamiltonian as $H=H^{0}+H^{1}$, where $H^{0}$ is the free Hamiltonian and $H^{1}$ characterizes the interaction. The interaction picture is then defined by letting the evolution of the operators be implemented by $H^{0}$ and the evolution of the states be implemented by $H^{1}$. It is connected to the Schrödinger picture by the transformations, $O_{I}(t)=e^{i H_{S}^{0} t} O_{S} e^{-i H_{S}^{0} t}$, and, $\psi_{I}(t)=e^{i H_{S}^{0} t} \psi_{S}(t)$. All three pictures agree at $t=0$, as $\psi_{I}(0)=\psi_{S}(O)=\psi_{H}$ and $O_{I}(0)=O_{H}(0)=O_{S}$.

These relations allow for the perturbative expansion of the time evolution operator which is defined by the relation $\psi\left(t_{1}\right)=U\left(t_{1}, t_{0}\right) \psi_{I}\left(t_{0}\right)$. Using the transformations connecting the pictures it can be shown that:

$$
U\left(t, t_{0}\right)=\sum_{n=0}^{\infty} \frac{(-i)^{n}}{n !} \int_{t_{0}}^{t} d t_{1} \ldots \int_{t_{0}}^{t} d t_{n} T\left(H_{I}^{1}\left(t_{1}\right) \ldots H_{I}^{1}\left(t_{n}\right)\right)
$$

\footnotetext{
${ }^{4}$ Throughout, the subscripts, $H$, and, $S$, denote the Heisenberg and Schrödinger picture, respectively. The Hamiltonian is the same in the Heisenberg and Schrödinger pictures and thus does not need a subscript.
} 
The $S$-matrix can then be defined in terms of the time evolution operator by,

$$
S_{j k}=\lim _{t_{2} \rightarrow \infty} \lim _{t_{1} \rightarrow-\infty}\left\langle\phi_{k}\left|U\left(t_{2}, t_{1}\right)\right| \phi_{j}\right\rangle,
$$

and thus, inserting the expansion for the time evolution operator yields the Dyson expansion for the $S$-matrix:

$$
S=\sum_{n=0}^{\infty} \frac{(-i)^{n}}{n !} \int_{-\infty}^{\infty} d t_{1} \ldots \int_{-\infty}^{\infty} d t_{n} T\left(H_{I}^{1}\left(t_{1}\right) \ldots H_{I}^{1}\left(t_{n}\right)\right)
$$

where the time ordered product rearranges the operators in the order of descending time argument. In general, $H_{I}^{1}$ is a product of free field operators describing the interaction between the fields. Evaluating the time ordered product of these products of field operators in the Dyson expansion can be simplified through an application of Wick's theorem. This theorem allows for the time ordered products in the expansion to be rewritten as a sum of contracted normal products, which are vacuum expectation values of time ordered interaction picture field operators. ${ }^{5}$ This technique allows for the perturbative evaluation of S-matrix elements for processes involving particular initial and final states. The interaction picture is essential for this perturbative evaluation because for $t= \pm \infty$, in the interaction picture the Hilbert space representation is simply the Fock representation for the free field. This makes it possible to explicitly calculate vacuum expectation values of products of interaction picture field operators. ${ }^{6}$

There are three primary obstacles to the well-definedness of this approach to the perturbative evaluation of field theoretic quantities, only one of which is related to Haag's theorem. ${ }^{7}$ The first two problems with the perturbative evaluation of Equation (4) come from the presence of ultraviolet and infrared divergences, respectively. Both types of divergences render individual terms in the sum infinite and thus the whole expression ill-defined. There are techniques for isolating and controlling these divergences. These methods, and how they restore the validity of perturbative evaluation of Equation (4) will be discussed in Section 4. Of the three obstacles to assigning meaning to the expression for the S-matrix, only the presence of infrared divergences is related to Haag's theorem. This class of divergences prevents the establishment of a global unitary transformation between the free and interacting fields, a critical assumption required for the establishment of the interaction picture. The third problem is that one is considering the sum of an infinite set of terms and it must be determined whether or not that sum converges.

\footnotetext{
${ }^{5}$ A detailed explanation can be found in, for example, (Greiner and Reinhardt 1996).

${ }^{6}$ This is not clear in the other pictures because one does not have an explicit representation of the field operators at asymptotic times.

${ }^{7} \mathrm{~A}$ clear discussion of all three problems can be found in (Haag 1992, pp. 70-71).
} 
There is reason to think that in empirically interesting models it does not. ${ }^{8}$ The final section of this paper explains an approach to understanding the meaningfulness of perturbation theory in face of this third problem.

Earman and Fraser provide a clear exposition of Haag's original argument (Haag 1955) and explain how Hall and Wightman (Hall and Wightman 1957) generalized the theorem. ${ }^{9}$ My aim here is to review some of the standard assumptions that go into the proof of the theorem and to show how the theorem undermines the existence of a global unitary transformation connecting the free and interacting fields. As Earman and Fraser correctly note, all of the assumptions required for the proof of the theorem are adopted in the approach to scattering theory based on the interaction picture. Many of these assumptions are also taken as axioms in the Wightman formalism for quantum field theory. ${ }^{10}$ Others are introduced specifically for the construction of the interaction picture for the perturbative evaluation of observables. The Wightman formalism consists of a set of statements about the properties of a collection of vacuum expectation values for a theory which together exhaust its physical content. They capture physical principles that are assumed to obtain for the objects described by the perturbative evaluation of field theoretic quantities. As Duncan explains, the proof can be understood as proceeding in two stages (Duncan 2012, p. 366). In the first stage it is shown that if two collections of field operators are globally unitarily equivalent, then the vacuum expectation values of products of those field operators at equal times must be identical. The second step is to show that this equality extends to arbitrary spacetime arguments of the fields. An application of the Wightman reconstruction theorem then ensures that the conclusion for field theories characterized in terms of vacuum expectation values also applies to field theories characterized in terms of operators acting on a Hilbert space.

Consider two neutral scalar fields $\phi_{j}, j=1,2$, with conjugate momenta $\pi_{j}$, where for each $j,\left(\phi_{j}, \pi_{j}\right)$ is an irreducible representation of the equal time canonical commutation relations,

$$
\begin{aligned}
& {\left[\phi_{j}(\vec{x}, t), \pi_{j}\left(\vec{x}^{\prime}, t\right)\right]=i \delta\left(\vec{x}-\vec{x}^{\prime}\right) j=1,2} \\
& {\left[\phi_{j}(\vec{x}, t), \phi_{j}\left(\vec{x}^{\prime}, t\right)\right]=\left[\pi_{j}(\vec{x}, t), \pi_{j}\left(\vec{x}^{\prime}, t\right)\right]=0 .}
\end{aligned}
$$

\footnotetext{
${ }^{8}$ There are arguments going back to (Dyson 1952) that suggest that the expansion in fact diverges in empirically interesting models. This has been confirmed rigorously in some simplified models.

${ }^{9}$ Haag's original version of the theorem fails to be fully general since it restricts attention to a particular class of Hamiltonians. The generalization due to Hall and Wightman closes this gap by extending Haag's result to cover all Hamiltonians. Additional helpful exposition can be found in (Duncan 2012).

${ }^{10}$ For the details of this approach see, for example, (Streater and Wightman 1964, pp. 96-102).
} 
Suppose further that the Euclidean transformations consisting of translations, $\vec{a}$, and rotations, $R$, are implemented by unitary operators $U_{j}(\vec{a}, R)$,

$$
\begin{gathered}
U_{j}(\vec{a}, R) \phi_{j}(\vec{x}, t) U_{j}^{-1}(\vec{a}, R)=\phi_{j}(R \vec{x}+\vec{a}, t) \\
U_{j}(\vec{a}, R) \pi_{j}(\vec{x}, t) U_{j}^{-1}(\vec{a}, R)=\pi_{j}(R \vec{x}+\vec{a}, t) .
\end{gathered}
$$

These are standard assumptions used in perturbative calculations and in the Wightman formalism. Finally suppose that at some time $t$ the fields are related by a unitary transformation $V(t)$,

$$
\phi_{2}(\vec{x}, t)=V(t) \phi_{1}(\vec{x}, t) V^{-1}(t), \quad \pi_{2}(\vec{x}, t)=V(t) \pi_{1}(\vec{x}, t) V^{-1}(t) .
$$

This is an assumption necessary for the construction of the interaction picture. These assumptions are sufficient to show that if there are unique normalizable Euclidean invariant states $\left|0_{j}\right\rangle,{ }^{11}$ then they must be related by, $c\left|0_{2}\right\rangle=V(t)\left|0_{1}\right\rangle$ where $|c|= \pm 1$. From this, the equality of the vacuum expectation values for products of equal time field operators follows directly. ${ }^{12}$ The extension of this equality to arbitrary spacetime arguments requires additional assumptions. Critically, the extension requires the full Poincaré invariance of the theory. Specifically, if $(\vec{a}, \Lambda)$ are Poincaré transformations implemented by the unitary operators $T_{j}(\vec{a}, \Lambda)$, then the fields transform as,

$$
T_{j}(\vec{a}, \Lambda) \phi_{j}(x)=\phi_{j}(\Lambda \vec{x}+\vec{a})
$$

and the $\left|0_{j}\right\rangle$ satisfy,

$$
T_{j}(a, \Lambda)\left|0_{j}\right\rangle=\left|0_{j}\right\rangle .
$$

The content of Hall and Wightman's generalization of Haag's argument is that on these assumptions, if $\phi_{1}$ is a free field then its vacuum expectation values are equal to those of $\phi_{2}$. This entails that they will also agree on all of their S-matrix elements.

Another way to state the content of the theorem is that if one assumes that the fields belong to the same Hilbert space representation, then if one of the fields is free, they are both free. It follows that free and interacting fields cannot belong to the same Hilbert space representation, an assumption on which the perturbative evaluation of field theoretic quantities in the interaction picture is predicated. For this reason, Haag's theorem undermines the approach to scattering theory based on the interaction picture in any the-

\footnotetext{
${ }^{11}$ Earman and Fraser note that this assumption follows from the classification of representations of the inhomogeneous Lorentz group (Earman and Fraser 2006, pp. 321-322).

${ }^{12}$ The details of the calculation are given in (Duncan 2012, pp. 367-368)
} 
ory satisfying the conditions of the theorem. Earman and Fraser claim that "... the problem brought to light by Haag's theorem is not directly related to the employment of perturbation theory as an approximation method; all of the assumptions of [Haag's] theorem are embraced before the perturbation series is even introduced" (Earman and Fraser 2006, p. 322). This is a point which merits further clarification. They are correct that the theorem is not concerned with the expansion of field theoretic quantities in a power series in general. What Haag's theorem undermines is precisely the pertubative evaluation of field theoretic observables in the interaction picture in particular. This is undermined by the theorem because the strategy that this method adopts for perturbative evaluation of observables requires the existence of a global unitary connecting the free and interacting fields that the theorem shows not to exist.

The Hall and Wightman generalization of the theorem holds for any pair of neutral scalar fields fields and any Hamiltonian satisfying the conditions of the theorem. In order to determine whether more physically relevant theories are plagued by an analogous result requires determining whether or not the result applies in the case of theories involving higher spin fields and in theories that couple different kinds of fields together. Generalizations of the theorem show that the interaction picture does not exist in essentially all cases in which the free and interacting Hamiltonians are defined on a continuum spacetime with the full Poincaré group as its spacetime symmetries and differ nontrivially. For the case of uncharged scalar fields, this level of generality is already present in the Hall-Wightman version of the theorem introduced here. Duncan has argued that as the complexity of the interaction in a theory grows, it is increasingly likely that there will fail to be unitary transformations connecting the Fock states of the free and interacting theories, and thus when more physically relevant interactions are considered, there is good reason to expect that an analog of Haag's theorem will obtain. ${ }^{13}$ For this reason, the theorem seems to show that empirical adequacy claims based on interaction picture calculations are unreliable.

3. Earman and Fraser on the success of scattering theory. This section considers how Earman and Fraser attempt to explain the success of scattering theory despite Haag's theorem. Their two part explanation appeals to techniques from axiomatic and constructive field theory. More specifically, they appeal to Haag-Ruelle scattering theory and theorems which establish the existence of local unitary equivalence between free and interacting theories. It should be made clear that they do not present their explanation as a full answer to the question of why the interaction picture and perturbation theory work. Instead they claim to "point to what [they] believe is a critical

\footnotetext{
${ }^{13}$ For a more detailed discussion of the generalization of the theorem see (Duncan 2012, pp. 363-369).
} 
piece in the overall scheme" (Earman and Fraser 2006, p. 322), and later they claim to have "indicated one route to such an explanation" (Earman and Fraser 2006, p. 333). They are not explicit about what, in their view, is missing from their account. This section explicitly identifies a critical respect in which their explanation of the success of perturbative calculations in scattering theory is deficient.

The first part of Earman and Fraser's explanation relies on the fact that Haag's theorem spoils global unitary equivalence, but it does not necessarily rule out local unitary equivalence. In some cases local unitary equivalence can be established, and they claim that when this is the case it underwrites a "... a perfectly good sense in which the interaction picture and perturbation theory do work ... at least for physical quantities that matter for explaining experimental outcomes" (Earman and Fraser 2006, pp. 323-324). What they seem to have in mind is that what actually get measured are observables in localized spacetime regions. To illustrate how this explanation works they consider the example of a theory of two free scalar fields with different masses, $\phi_{m_{1}}$, and $\phi_{m_{2}}$, with the masses related by $m_{2}=m_{1}+\delta m$. In this case they note that local unitary equivalence can be rigorously established. ${ }^{14}$

For Earman and Fraser, this shows why perturbation theory can be used to explain the results of experiments on local observables using such a theory. They note that this solution to the problem is also a viable one in the case of $\left(\phi^{4}\right)_{2}$ theory, the theory of a self-interacting neutral scalar field in one space and one time dimension. While this is a more physically relevant interaction than the mass shift in their first example, it is highly simplified in that it is defined in reduced spacetime dimension. Many of the models which have successfully been constructed are defined in fewer than four spacetime dimensions because such models tend to have less severe divergences. Earman and Fraser explain that since $\left(\phi^{4}\right)_{2}$ theory does not have ultraviolet divergences and the restriction to bounded regions of spacetime involved in the definition of local unitary equivalence removes the possibility of infrared divergences, it was to be expected that local unitary equivalence could be established in this case. They then note that in higher spacetime dimensions the ultraviolet problems become worse, the full power of renormalization methods are required for the theory to be well-defined, and local unitary equivalence is spoiled. Earman and Fraser then declare that "... Haag's theorem is not responsible for the problems created by ultraviolet divergences, so solving them is beyond the scope of this paper" (Earman and Fraser 2006, p. 323). It is true that Haag's theorem captures a mathematical problem associated with infrared and not

\footnotetext{
${ }^{14}$ The precise sense of local unitary equivalence that they appeal to is the following one: "Given any bounded region $B \subset \mathbb{R}^{3}$ and the free fields $\phi_{m_{1}}, \pi_{m_{1}}$ and $\phi_{m_{2}}, \pi_{m_{2}}$ acting on the respective Hilbert spaces, $\mathcal{H}_{1}$ and $\mathcal{H}_{2}$, there is a unitary map $V_{B}: \mathcal{H}_{1} \rightarrow \mathcal{H}_{2}$ such that $V_{B} \phi_{m_{1}}(f) V_{B}^{-1}=\phi_{m_{2}}(f)$ and $V_{B} \pi_{m_{1}}(f) V_{B}^{-1}=\pi_{m_{2}}(f)$ for all suitable test functions $f$ with support in $B^{\prime \prime}$ (Reed and Simon 1975, p. 329).
} 
ultraviolet divergences. However, in the next section I will argue that, in order to understand the success of the perturbative evaluation of scattering matrix elements, all three mathematical problems with Equation (4) introduced above need to be resolved. In this sense, the solution to the problem of ultraviolet divergences does play a role in restoring the validity of the interaction picture. What should be noted at this point is that the solution of the problem by appeal to local unitary equivalence is only demonstrably valid in the case of a handful of simplified models, and not in the field theories in four-dimensional Minkowski space that make up the Standard Model. Since such realistic theories all contain ultraviolet divergences, there is good reason to expect that local unitary equivalence will be spoiled in those cases as well.

The second part of Earman and Fraser's explanation is an appeal to the formalism for scattering theory developed by Haag and Ruelle. ${ }^{15}$ This framework begins by assuming that the theory in question satisfies the Wightman axioms and then stipulates that they also satisfy an additional condition on the spectrum of the Hamiltonian to ensure the existence of a mass gap. ${ }^{16}$ The central idea of their framework is to rigorously construct the Hilbert spaces $\mathcal{H}_{\text {in }}$ and $\mathcal{H}_{\text {out }}$ spanned by the states before and after the scattering using elements from the full Hilbert space, $\mathcal{H}$, in the asymptotic limit where $t \rightarrow \pm \infty$. Earman and Fraser note that "This formalism is not subject to Haag's theorem because - unlike the interaction picture - it neither posits nor entails the existence of a unitary transformation connecting $\mathcal{H}\left(\right.$ or $\mathcal{H}_{\text {in }}$ or $\mathcal{H}_{\text {out }}$ ) to $\mathcal{H}_{F}$ that relates the interacting field to a free field" (Earman and Fraser 2006, p. 326). This approach thus seems to afford the possibility of circumventing the problem raised by Haag's theorem entirely. ${ }^{17}$

With respect to the interpretive significance of this formalism Earman and Fraser note that "... the Haag-Ruelle approach shows how to maneuver around [Haag's theorem] to obtain in QFT analogues for most of the significant features of ordinary scattering theory" (Earman and Fraser 2006, p. 326). They do not raise any particular features as examples, but it is certainly true that for the models to which the Haag-Ruelle theory applies, the formalism shows how to obtain many of the features of standard scattering theory without running afoul of Haag's theorem. One of the central benefits of the constructive approach to scattering theory is that it goes even further and affords explanations for features of the perturbative treatment of the the-

\footnotetext{
15 (Haag 1958; Ruelle 1962)

${ }^{16}$ That is, it is required that the operator $P^{2}=P_{\mu} P^{\mu}$ has an isolated eigenvalue $m^{2}>0$, corresponding to the single particle states, and the remaining part of the spectrum is continuous, beginning at $(2 m)^{2}$. See, for example, (Iagolnitzer 1993, p. 72) for further discussion.

${ }^{17}$ It is not unique in this respect. As Bain has emphasized, the LSZ formalism is also able to escape the force of Haag's result in a related way (Bain 2000).
} 
ory that typically must be taken as assumptions. ${ }^{18}$ Moreover, since explicit models can be constructed, it is clear that the Haag-Ruelle theory is based on a mathematically consistent framework.

This part of Earman and Fraser's explanation of the success of scattering theory is limited in very much the same way as the first part. In particular, it can only be shown to be valid in certain simplified models ${ }^{19}$ and it is not clear that the explanatory significance can be exported from those models to cases of experimental interest. There is no known model of a field theory with local gauge symmetry defined in four-dimensional Minkowski space that satisfies the Wightman axioms and exhibits a mass gap. The field theories that make up the Standard Model are all, however, local gauge theories. This undermines the ability of the Haag-Ruelle theory to explain the success of scattering theory in realistic theories in a straightforward way.

At this stage one might object that none of the discussion up to this point rules out the possibility that more physically relevant theories will be shown to satisfy the Wightman axioms or some modified set of axioms characterizing the non-perturbative content of the theory. This is certainly an open possibility, and if it was accomplished then empirically adequate theories could be treated using Haag-Ruelle theory or some close analog for the new axiomatization. Moreover, if this were achieved then Earman and Fraser would have provided an adequate explanation for how scattering theory can be done in a mathematically consistent manner despite Haag's theorem. However, there remains a clear sense in which their explanation is deficient as a response to the question of why scattering theory works despite Haag's theorem.

In order to show that empirical adequacy claims for particular quantum field theories are safe from the theorem, it must be shown that the theoretical predictions that are actually used to match with data are not affected by Haag's result. For Earman and Fraser's response to the theorem to be helpful for this task, it would need to be the case that the theoretical predictions are calculated using the constructive formalism to which they appeal. I have already noted the reason why this cannot be the case: the techniques they appeal to are not demonstrably applicable in the cases of the theories of experimental interest. In some cases, theoretical predictions have been cal-

\footnotetext{
${ }^{18}$ These features include the presence of the clustering property. While in perturbative treatments of the theory this property is simply assumed as a phenomenological constraint which rules out dependence between far separated scattering experiments, in the Haag-Ruelle formalism it can be recovered as a consequence of the Wightman framework for quantum field theory. Another such feature is the existence of the asymptotic states. Whereas in the LSZ formalism asymptotic completeness is assumed, this feature is recovered as a theorem in the Haag-Ruelle formalism. See (Strocchi 2013, p. 123) for further discussion.

${ }^{19}$ The models in which the Haag-Ruelle theory can be shown to apply include weakly

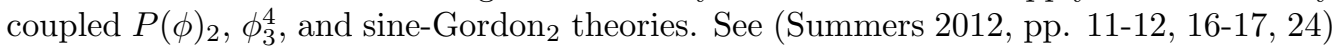
for discussion.
} 
culated using the interaction picture formalism whose validity Haag's result casts into doubt. Other techniques for obtaining theoretical predictions are also used, but they are not the constructive techniques to which Earman and Fraser appeal. For these reasons I claim that Earman and Fraser have not provided an explanation of why empirical adequacy claims for quantum field theory are not undermined by the theorem. As a result, a complete explanation for why scattering theory works is still lacking. In the next section I argue that the calculational techniques employed in the calculation of realistic experimental observables render some of the assumptions of Haag's theorem false. It is on this basis that one can conclude that there is no reason to expect that Haag's theorem undermines empirical adequacy claims.

4. Haag's theorem and empirical adequacy. If the theoretical calculations that are used to compare with experiments were in fact shown to be invalid by Haag's theorem, it would undermine much of the direct evidence for the Standard Model. The concern about inconsistency raised by the theorem can be resolved by looking to the techniques that are used in the calculations that are compared with experiments. In some calculations the interaction picture is not used. In other calculations regularization and renormalization techniques render some of the assumptions of Haag's theorem false and thus show how it is possible to obtain meaningful answers using the interaction picture formalism. In both cases I submit that there is no stage in the calculation at which the quantities in question are ill-defined because of Haag's theorem and it is this fact that grounds the reliability of empirical adequacy claims in the face of the theorem.

In theories with strong coupling, such as quantum chromodynamics, the interaction picture formalism is not used. Since the coupling is strong, the parameter in which one is expanding is large and perturbation theory cannot be expected to give meaningful answers. In this case a different approach to generating predictions is necessary. Strongly coupled theories can be regularized by placement on a Euclidean lattice, and contributions to expressions for physical observables can be approximated numerically. Realistic modern experiments frequently have contributions from quantum chromodynamic processes, and thus empirical adequacy claims are dependent on these calculational techniques. Of course, in this case, the interaction picture simply is not employed at any point in the calculation, and Haag's theorem provides no obstacle to the calculation of experimental observables.

There are, however, cases in which the interaction picture is used to calculate physical observables. This is the context in which Haag's theorem raises a legitimate concern about empirical adequacy claims. The interaction picture was first introduced by Schwinger in (Schwinger 1948b). One of the motivations for its introduction was to facilitate the calculation of the anomalous magnetic moment of the electron and thus to provide a critical test of 
the empirical adequacy of quantum electrodynamics. Since its introduction, the perturbative evaluation of vacuum expectation values and S-matrix elements for weakly coupled theories like quantum electrodynamics has relied on the interaction picture formalism. An adequate explanation of the success of scattering theory must show why such calculations give values that match empirical data despite Haag's theorem.

Rendering the perturbative evaluation of S-matrix elements for interacting quantum field theories well-defined requires that all three problems with Equation (4) by addressed. Regularization and renormalization techniques are used to isolate and control the infrared and ultraviolet divergences in the theory. There are several different regularization schemes which can be used to control ultraviolet divergences. The simplest example of such an ultraviolet regularization is the imposition of a short distance, or equivalently large momentum, cutoff. ${ }^{20}$ When long distance cutoff is also imposed to control the infrared divergences, the theory is reduced to a finite number of degrees of freedom. Once a regularization is in place, the theory can be renormalized. At the end of a calculation the regularizations can be removed by taking the limit where the spacetime approaches continuous and infinite Minkowski space, thus restoring the full symmetry properties of the theory.

The full regularization that is imposed to control ultraviolet and infrared divergences breaks the Poincaré invariance of the theory. Recall that this an essential assumption required to prove Haag's theorem. In the fully regularized theory, each contribution to the perturbative expansion is thus welldefined when it is evaluated. With the regularization in place the perturbative expansion for the S-matrix elements defined by Equation (4) can proceed order by order. The number of terms that must be summed to obtain the contribution from each order grows rapidly, and thus the state of the art only allows for perturbation theory calculations at a few orders for most important observables. The essential thing to note is that what gets compared to experimental data is the sum of the first few terms of the expansion. Since all of the terms in the sum are well-defined when they are calculated, there simply is no problem caused by Haag's theorem. The perspective that I am advocating has recently been argued for by Duncan. ${ }^{21}$ He claims that "...the proper response to Haag's theorem is simply a frank admission that the same regularizations needed to make proper mathematical sense of the dynamics of an interacting field theory at each stage of a perturbative calculation will do double duty in restoring the applicability of the interaction picture at in-

${ }^{20}$ The details of regularization and renormalization techniques can be found in most standard texts on quantum field theory. For a more comprehensive presentation see (Collins 1984).

${ }^{21}$ Butterfield's review of Duncan's book draws attention to the importance of this argument (Butterfield 2015). 
termediate stages of the calculation" (Duncan 2012, p. 370) ${ }^{22}$ I agree with Duncan that the regularizations used to control the ultraviolet and infrared divergences are what preserves the reliability of perturbative calculations in the face of Haag's theorem, but there is one futher concern that must be addressed.

One might worry that this resolution to the problem is not completely general. In particular, there is more than one approach to regularizing and renormalizing field theories. Moreover, each method has different effects on the symmetries of the theory. Some methods break Poincaré invariance and others break gauge invariance. Which technique gets used for a particular calculation depends on which properties of the theory one wants to preserve. Thus, to put the worry precisely, one might wonder if some of these techniques leave the full Poincaré symmetry intact. ${ }^{23}$ If this were the case then it would seem that such calculations are still subject to Haag's theorem. Consider, for example, the technique of dimensional regularization. Rather than imposing cutoffs one continues the spacetime dimension to $4-\epsilon$. This has benefit of preserving gauge invariance. The question of Poincaré invariance is more sensitive as the exact spacetime symmetries of Minkowski space are not restored until the dimension is continued back to 4 . However, for the purposes of my argument what is critical to note is that dimensional regularization also affords the capability to control infrared divergences. ${ }^{24}$ In order to achieve sensible perturbative results using the dimensional regularization, the infrared divergences must be addressed using such techniques.

In practice, empirical adequacy claims often involve sums of contributions to different orders obtained using different regularization techniques. Consider, for example, the calculation of the anomalous magnetic moment of the electron. The best theoretical calculation of this observable matches experimental data to more than 10 decimal places. The first order contribution to this quantity was originally calculated by Schwinger (Schwinger 1949). During the process of the calculation, he encounters an infrared divergence. To control it he introduces a minimum wave number for the photons in the theory, which is equivalent to the imposition of a maximum wavelength and thus a long distance cutoff. ${ }^{25}$ Since this quantity provides such a critical precision test of the theory, significant effort has been dedicated to calculating additional orders of perturbation theory beyond the leading term. ${ }^{26}$ Some intermediate orders can be calculated analytically, but this analytic evaluation

\footnotetext{
${ }^{22} \mathrm{~A}$ similar perspective can also be found in (Strocchi 2013, p. 52).

${ }^{23}$ I am grateful to Kerry McKenzie for pressing me on this point.

${ }^{24}$ For the details of this approach see (Gastmans and Meuldermans 1973; Marciano and Sirlin 1975).

${ }^{25}$ See equation 1.107 (Schwinger 1949, p. 801).

${ }^{26}$ (Kinoshita 1990; Roskies, Levine, and Remiddi 1990; Aoyama, Hayakawa, Kinoshita, and Nio 2012; Kinoshita 2014)
} 
requires regularizations that break Poincaré invariance. The highest orders require the computation of a very large number of complicated terms and must be computed numerically. This of course requires that the theory be reduced to a finite number of degrees of freedom and so again Haag's theorem is rendered inapplicable.

The real difficulty raised by Haag's theorem then, is to understand why contributions from the first few orders of perturbation theory give empirically adequate results, even though when the full symmetries are restored by taking the infinite volume limit and removing the ultraviolet regularization, the formalism used to obtain those results becomes ill-defined. The best available explanation of this fact is that we calculate observables that are insensitive to the removal of the infrared cutoff, the so-called infrared safe observables. To Earman and Fraser's collections of morals that should be gleaned from the theorem, my analysis suggests a fourth: Haag's theorem complicates the relationship between the perturbative content of the theory and our best available characterization of its non-perturbative structure. This is important moral, but it is not unique in this respect. The question of how well pertubative data constrains non-perturbative structure is a very general one, and about which much information is available from sources other than Haag's theorem. Even in quantum chromodynamics, where the interaction picture is not used and there is no problem with Haag's theorem, a similar question arises. Results are calculated on a lattice and give empirically adequate results. However, the full continuum theory has not been shown to be a model of the axioms that are believed to characterize the structure of the theory.

5. Conclusion. I have argued that empirical adequacy claims are not undermined by Haag's theorem because the regularizations and renormalization required to give clear meaning to the perturbative evaluation of vacuum expectation values and S-matrix elements also quell the problems associated with the infrared divergences implicated in Haag's theorem. The constructive approach to field theory takes as its starting point physical assumptions that are believed to obtain in the empirically adequate models that can currently only be treated perturbatively. According to this perspective, what the Wightman axioms amount to is a collection of the basic physical properties that need to be satisfied in the continuum and infinite volume limits in order to have what can properly be counted as a relativistic quantum field theory. However, as I have stressed above, the theories of the Standard Model cannot be shown to satisfy the axioms. Obtaining numerical information from them for comparison with experiment requires that they be regularized in ways that render some of the conclusions that can be reached in the unregularized theory, including Haag's theorem, inapplicable. It follows that achieving a complete understanding of why scattering theory does work requires a resolution to the tension between the mathematical characterization 
of the non-perturbative structure of the theory and the techniques that are required to obtain successful empirical predictions using that structure. Haag's theorem should thus be understood as providing a constraint on the nature of the relation between the perturbative expansion and non-perturbative characterizations of the content of the theory.

Reactions to Haag's theorem are illustrative of a general tension which exists among much of the literature that attempts to address the interpretation of quantum field theory. It is not obvious what the rigorous models have to do with the actual world because they are defined in a spacetime with dimension other than four or without realistic interactions. At the same time, the corrections to the mathematical formalism required to render the expressions characterizing empirically relevant models well-defined seem to correspond to physically substantive changes according to standard approaches to interpretation. There are two ways that this tension might be resolved. First, it could be that further work will lead to existence proofs for more physically relevant models. If this were achieved then the Haag-Ruelle formalism that Earman and Fraser appeal to could underwrite the success of scattering theory directly. Much of the literature appraising the philosophical significance of quantum field theory seems to be predicated on the hope that this goal will be achieved. In fact, some authors seem to think that this is a necessary condition for quantum field theory to be a foundationally respectable theory, and that absent such a development claims about perturbative field theory are mathematically unintelligible. However, there is no assurance that physically relevant theories are in fact models of the axioms. If they are not, then one could appropriately view the inability to construct models of the axioms as a source of physical information. In this case, the success of scattering theory would need to be accounted for in a more elaborate way.

Further evidence that a more elaborate account is necessary comes from attempts to address the third, and in my opinion the most important, problem with the perturbative expansion. Even though the first few terms in the expansion give a result which agrees closely with experiment, when the contributions from higher orders of perturbation theory are included, the series goes on to diverge. The divergence in question is independent from the ultraviolet and infrared divergences that are controlled with regularization and renormalization. The perturbation series itself diverges, even once the theory has been renormalized to render each term in the expansion finite. An explanation of the success of scattering theory should also account for the fact that taking the first few terms of what are widely believed to be divergent expansions give such remarkably accurate results. An explanation of this fact is provided by the conjecture that empirically successful perturbative expansions are asymptotic to exact solutions of a theory that generates them. 
Many non-perturbative structures can yield the same asymptotic expansion. Thus, the conjecture that perturbation theory in quantum electrodynamics yields asymptotic expansions does not uniquely fix what non-perturbative structure lies behind the empirical success of the theory. The more elaborate account I have in mind must address how well perturbative data can constrain the non-perturbative structure of the theory, as well as the fact that the empirical information that we glean from experiments seems to exhibit a level of insensitivity to the exact non-perturbative structure. The analysis of this paper shows that Haag's theorem does not undermine empirical adequacy claims, and it also shows that the theorem does not undermine the use of perturbation theory as a guide to determining non-perturbative structure.

6. Acknowledgements. I am thankful to John Earman, John Norton, Giovanni Valente, and audiences in Irvine and Chicago for helpful comments on this paper. I am especially grateful to Bob Batterman, Gordon Belot, Doreen Fraser, and Laura Ruetsche for detailed criticism of an earlier draft. 


\section{References}

Aoyama, T., M. Hayakawa, T. Kinoshita, and M. Nio (2012). Quantum electrodynamics calculation of lepton anomalous magnetic moments: Numerical approach to the perturbation theory of QED. PTEP 2012, $01 \mathrm{~A} 107$.

Bain, J. (2000). Against Particle/Field Duality: Asymptotic Particle States and Interpolating Fields in Interacting QFT (Or: Who's Afraid of Haag's Theorem?). Erkenntnis 53(3), 375-406.

Barton, G. (1963). Introduction to advanced field theory. Interscience Publishers.

Bloch, F. and A. Nordsieck (1937). Note on the Radiation Field of the electron. Phys.Rev. 52, 54-59.

Butterfield, J. (2015). Review: Anthony Duncan. The Conceptual Framework of Quantum Field Theory. Philosophy of Science 82(2), 326-330.

Collins, J. C. (1984). Renormalization: An Introduction to Renormalization, the Renormalization Group and the Operator-Product Expansion. Cambridge University Press.

Duncan, A. (2012). The Conceptual Framework of Quantum Field Theory. Oxford University Press.

Dyson, F. (1952). Divergence of perturbation theory in quantum electrodynamics. Phys.Rev. 85, 631-632.

Earman, J. and D. Fraser (2006). Haag's Theorem and its Implications for the Foundations of Quantum Field Theory. Erkenntnis 64 (3), 305-344.

Gastmans, R. and R. Meuldermans (1973). Dimensional regularization of the infrared problem. Nucl.Phys. B63, 277-284.

Greiner, W. and J. Reinhardt (1996). Field quantization. Springer.

Haag, R. (1955). On quantum field theories. Det Kongelige Danske Videnskabernes Selskab, Matematisk-fysiske Meddelelser 29(12), 1-37.

Haag, R. (1958). Quantum Field Theories with Composite Particles and Asymptotic Conditions. Physical Review 112(2), 669-673.

Haag, R. (1992). Local Quantum Physics. Fields, Particles, Algebras. Springer-Verlag.

Hall, D. and A. Wightman (1957). A theorem on invariant analytic functions with applications to relativistic quantum field theory. Det Kongelige Danske Videnskabernes Selskab, Matematisk-fysiske Meddelelser 31(5), 1-41. 
Huggett, N. and R. Weingard (1994). Interpretations of quantum field theory. Philosophy of Science 61(3), 370-388.

Iagolnitzer, D. (1993). Scattering in quantum field theories: the axiomatic and constructive approaches. Princeton University Press.

Kinoshita, T. (1962). Mass singularities of Feynman amplitudes. J.Math.Phys. 3, 650-677.

Kinoshita, T. (1990). Theory of the anomalous magnetic moment of the electron - numerical approach. Adv.Ser.Direct.High Energy Phys. 7, $218-321$.

Kinoshita, T. (2014). Tenth-order QED contribution to the electron g-2 and high precision test of quantum electrodynamics. International Journal of Modern Physics A29, 1430003.

Lee, T. and M. Nauenberg (1964). Degenerate Systems and Mass Singularities. Phys.Rev. 133, B1549-B1562.

Lupher, T. (2005). Who Proved Haag's Theorem? International Journal of Theoretical Physics 44(11), 1995-2005.

Marciano, W. and A. Sirlin (1975). Dimensional Regularization of Infrared Divergences. Nucl.Phys. B88, 86.

McCabe, G. (2007). The structure and interpretation of the Standard Model.

Muta, T. (2010). Foundations of Quantum Chromodynamics: An Introduction to Perturbative Methods in Gauge Theories, (3rd ed.).

Reed, M. and B. Simon (1975). Methods of Modern Mathematical Physics, Fourier Analysis, Self-Adjointness, vol. 2. Elsevier.

Roskies, R., M. J. Levine, and E. Remiddi (1990). Analytic evaluation of sixth order contributions to the electron's g factor. Advanced Series on Directions in High Energy Physics 7, 162-217.

Ruelle, D. (1962). On the Asymptotic Condition in Quantum Field Theory. Helvetica Physica Acta 35, 147-163.

Ruetsche, L. (2011). Interpreting Quantum Theories. Oxford University Press.

Schweber, S. (1994). QED and the men who made it: Dyson, Feynman, Schwinger, and Tomonaga.

Schwinger, J. S. (1948a). On Quantum electrodynamics and the magnetic moment of the electron. Phys.Rev. 73, 416-417.

Schwinger, J. S. (1948b). Quantum electrodynamics. I A covariant formulation. Phys.Rev. 74, 1439. 
Schwinger, J. S. (1949). Quantum electrodynamics. III: The electromagnetic properties of the electron: Radiative corrections to scattering. Phys.Rev. 76, 790-817.

Sklar, L. (2000). Theory and truth: philosophical critique within foundational science. Oxford University Press.

Streater, R. F. and A. S. Wightman (1964). PCT, Spin and Statistics, and All That. Princeton University Press.

Strocchi, F. (2013). An Introduction to Non-Perturbative Foundations of Quantum Field Theory. Oxford University Press.

Summers, S. J. (2012). A Perspective on Constructive Quantum Field Theory.

Teller, P. (1995). An interpretive introduction to quantum field theory. Princeton University Press. 